\title{
Per la "femmina balba"
}

\section{Paolo Cherchi}

Anche la "femmina balba," come tutte le femmine del mondo, ha avuto i suoi corteggiatori alletati non dalla promessa d'un languido sguardo dei suoi occhi guerci o dal perverso piacere di godere piú a lungo del suo fetore, o delle carezze dai suoi moncherini, ma solo dal desiderio di carpire il segreto dell'allegoria che si cela dietro la sua inusitata laidezza. Come ogni lettore ricorderà questa donna vive solo nella visione di Dante. La notte coglie il poeta al quarto girone; quindi, per la seconda volta nel Purgatorio, s'addormenta, trasmutando "il pensamento in sogno." All'alba, nel momento, cioè, in cui i sogni sono veraci,

mi venne in sogno una femmina balba, negli occhi guercia, e sovra i piè distorta,

con le man monche, e di colore scialba (XIX. 7-9)

Ma sotto lo sguardo del poeta questa femmina si trasforma in sirena che ammalia i marinai e che cantando si vanta d'aver volto "Ulisse del suo cammin vago": infatti chi "si ausa" con lei ne rimane del tutto appagato. Il suo canto non s'è ancora chiuso sull'ultima nota che una "donna santa e presta" appare al fianco di Dante e apostrofa Virgilio chiedendogli chi sia mai questa sirena. Quindi

L'altra prendea, e dinanzi l'apria

fendendo i drappi, e mostravami il ventre:

quel mi svegliò col puzzo che n'uscia

Questo sogno, come del resto gli altri due del Purgatorio, è chiaramente allegorico. La deformità della femmina, la sua metamorfosi e il richiamo ad Ulisse; l'intervento d'una donna non altrimenti identificata; l'operazione di strappare la camicia della femmina balba risultano fatti tutt'altro che perspicui nel loro signi- 
ficato, senza dire che la sequenza degli eventi è tale che solo il sogno può giustificarla. Sifatte circostanze avviano il processo ermeneutico, e, come si può immaginare, l'industriosità dei lettori di Dante non s'è risparmiata sudori ed elucubrazioni per veder chiaro nel sogno del poeta. ${ }^{1}$ Ma qui non si vuole entrare nella mischia. Questa nota aspira soltanto ad indicare una possibile filigrana che operava nella memoria di Dante quando creò quest'episodio.

Il primo ad indicare un modello per la femmina balba fu Giuseppe Toffanin. In un breve contributo apparso sul Giornale storico della letteratura italiana il Toffanin presentò un episodio ricavato dalle Vitae Patrum, episodio che ricorda la storia del figlio d'un santo padre, il quale sta per abbandonarsi "ad saeculum" perché non può piú resistere agli stimoli della carne. Il padre gli consiglia di raccogliersi in preghiera in un eremo. Il figlio obbedisce, e dopo venti giorni di raccoglimento vede

opus quoddam diabolicum venire super se; et stetit coram ipso velut mulier Aetiopissa, foetida et turpis aspectu, ita ut foetorem eius sufferre non posset; et abjeciebat eam a se. Et illa dicebat ei: Ego sum, quae in cordibus hominum dulcis appareo, sed propter oboedientiam tuam, et laborem quem sustines, non me permisit Deus seducere te sed innotui tibi foetorem meum. Ille autem surrexit. ${ }^{2}$

Il riscontro è suggestivo; tuttavia, come del resto nota il Toffanin, manca il particolare della donna santa e presta; manca - possiamo aggiungere - il particolare del "fendere i drappi" e, soprattutto, la fetida e turpe femmina non insiste nella sua opera di seduzione. Nondimeno l'indicazione del Toffanin rimane la migliore rispetto a proposte seriori. Una di queste avanza la candidatura delle "scenicae meretriculae," ovvero le Muse, le dolci sirene, che Filosofia della Consolatio Philosophiae caccia dalla vista di Boezio. ${ }^{3}$ Un'altra candidatura - avanzata dal Papparelli ${ }^{4}$ - proviene dalle pagine di Gregorio Nisseno.

A fianco di questi supposti modelli se ne vuol qui presentare un altro che - ma su questo giudicheranno i lettori - può essere un degno concorrente e può persino essere integrato con gli altri: dopo tutto al senso interno dell'imaginatio competeva il combinare immagini aventi provenienza diversa. Il testo che proponiamo non è ricavato da un trattato morale o edificante, ma da un fortunatissimo manuale di medicina di Bernardo di Gordon noto sotto il titolo abbreviato di Lilium medicinae. Il passo in questione si trova alla fine d'un capitolo dedicato all'amore hereos, all'amore, cioè, 
che è una vera e propria malattia causante un eccesso di umore malinconico. Il passo suggerisce un modo estremo di terapia, un modo al quale si deve ricorrere quando ogni altro rimedio fallisce:

Finaliter autem cum aliud consilium non habemus, imploremus auxilium et consilium vetularum, ut ipsi dehonestent et diffament quantum possunt. Ipsae enim habent artem sagacem ad hoc plus quam viri. Cum dicit Avicenna aliqui sunt qui gaudent in audiendo foetida et illicita. Quaeratur igitur vetula turpissima in aspectu cum magnis dentibus et barba et cum turpi et vili habitu; et quae portet subter gremium pannum menstruatum et adveniens philocapta quae incipiat dehonestare camisiam suam dicendo quantum est tignosa et ebriosa; et quae mingit in lecto; et quae est epileptica et impudica; et quae in corpore suo sunt excrescentiae enormes cum foetore anhelitus, et aliis omnibus enormibus in quibus vetulae sunt edoctae. $\mathrm{Si}$ autem ex hiis persuasionibus nolit dimittere, subito extrahat pannum menstruatum coram faciem, apostando dicendo clamando: talis est amica tua, talis. Et si ex hiis non dimiserit iam non est homo sed diabolus incarnatus. ${ }^{5}$

Dante conobbe questo passo? Mancando ogni esplicito riferimento all'opera di Bernardo Gordon tale conoscenza si può dedurre soltanto dalla qualità dei riscontri e dall'orientamento generale o specifico della cultura dantesca. Per quel che tocca il primo criterio metodologico, il particolare che rende cogente il riscontro è il "dehonestare camisam," ${ }^{\prime 6}$ che sarebbe difficile spiegare con l'appello ad una pura coincidenza. Se questa non è una fallace suggestione, si può allora arricchire tale riscontro con altri particolari: la compresenza di due donne; la puzza esalante dal ventre e il particolare del panno mestruato; l'effetto terapeutico del disinnamoramento. Si può aggiungere, inoltre, che il passo del Lilium viene dopo una discussione sulla semiotica e sull'eziologia della malattia d'amore; l'episodio della "femmina balba" viene dopo la discussione del fenomeno dell'amore: in entrambi i testi ricorrono motivi identici; ma ciò si deve spiegare con una fonte in comune che è, grosso modo, la teoria aristotelico-galenica della percezione e dell'attività dei sensi interni.

Quanto al secondo principio metodologico, esso ci offre elementi piú sicuri. È noto, infatti, che Dante ebbe interesse vivo e duraturo per la medicina, e ciò risulta non dal fatto che fu iscritto all'Arte dei medici e degli speziali, ma dalle numerose tracce di linguaggio medico presenti nelle sue opere. ${ }^{7}$ È normale, dunque, pensare che un tale interesse l'avrebbe portato alla lettura del $\mathrm{Li}$ lium che pubblicato nel 1305 ebbe una rapida e vasta diffusione. 
Qualora si trovasse poco convincente la proposta della "vetula turpissima" come modello per la "femmina balba," bisognerà ammettere che il passo qui riportato utilizzi un topos che affiora anche in Dante: pertanto sarebbe inutile cercare fonti precise. Non è un caso se possiamo rintracciare in parte la "femmina balba," la "foetida Aetiopissa" e la "vetula turpissima" in quella Beroe che Matteo di Vendôme ${ }^{8}$ costruí come modello artistico d'ogni vecchia che fuga ogni tentazione.

\section{University of Chicago}

\section{NOTES}

1 Fra i contributi piú persuasivi vanno segnalati i ripetuti interventi di G. Paparelli in parte sistemati nel suo volume Questioni dantesche (Napoli: Libreria Scientifica Editrice, 1967), pp. 181-213. Fra i contributi piú recenti ricorderò quello di D. Cervigni, "Demonic and Angelic Forces in Dante's Second Dream," in L'Alighieri 18 (1977), 29-40, con ricca bibliografia.

2 "La 'foetida Aetiopissa' e la 'femmina balba'," in GSLI, 77, 147-49 (la citazione è da p. 148), poi ristampato in Sette interpretazioni dantesche (Napoli: Libreria Scientifica Editrice, 1947), pp. 29-34.

3 G. Fabris, "Il secondo sogno di Dante nel Purgatorio," in Giornale Dantesco, 26 (1923), 104.

4 Op. cit., p. 184, n. 3.

5 Tabula pratice Gordoni dicte Lilium medicine Venezia, 1496), lib. Il, cap. XX "de amore qui hereos dicitur," ca. $71^{v}$. Nel citare ho risolto le abbreviature, restaurato i dittonghi e sono intervenuto nell'interpunzione. Il luogo d'Avicenna citato da Bernardo è sicuramente questo: "Etiam vetulae ad eum incitentur ut vituperent illud quod diligunt ipsi, et rememorentur eius dispositiones et narrent ei res aliquas de ipso vituperationes multas. Haec enim sunt ex eis quae sedant plurimum: quamvis sit ex eis quae alios consolentur. Et ex eis, quae ad illos iuvant est ut narrent vetulae istae formis eius quod diligitur cum similitudinibus foetidis et assimilentur membra faciei eius cum narrationibus horribilibus, et assiduent illud et perseverent in ipso. Haec enim sunt ipsarum opera et sunt prudentiores in hoc viris, nisi mollibus." Questo passo è ricavato dal Canon lib. III, fen. 1, tract. IV, cap. 23 (in Libri in re medica omnes [Venezia: apud Vincentium Valgrisium, 1564], p. 480). Non è improbabile che una ricerca piú ampia nella letteratura medica porti esempi simili di vecchie laide che operano il disinnamoramento e che si tratti, dunque, di terapia usitata di cui poi Dante si sarebbe ricordato nell'episodio della femmina balba.

6 Considerando il valore riflessivo di "suam" non ci dovrebbe esser dubbio sul fatto che la vecchia apra la propria camicia. Tuttavia è nota l'oscillazione dell'aggettivo possessivo suus in Francia, dove, fin dal sesto secolo venne spesso a sostituire eius (cfr. D. Norberg, Manuel practique de latin médiévale [Parigi: A. et J. Picard et C , 1968], p. 27 sg.). Se questo fosse il caso, l'episodio di Bernardo sarebbe ancora piú vicino a quello di Dante. Si ricorderà, inoltre, che il soggetto di "L'altra prendea" non è del tutto certo: alcuni commentatori antichi e moderni (Landino, Vellutello, Barbi e Sapegno) intendono Virgilio, altri (Ottimo, Benvenuto, Buti, Scartazzini-Vandelli) pensano alla "donna santa e presta": se in qualche modo il passo di Bernardo influí su Dante, esso farebbe propendere per la seconda spiegazione. 
7 Copiose indicazioni in questo senso si troveranno in B. Nardi, "L'amore e i medici medievali," in Studi in onore di A. Monteverdi (Modena: Società Tipografica Editrice Modenese, 1959), vol. 2, pp. 517-42; M. Mattioli, Dante e la medicina (Napoli: Edizioni Scientifiche Italiane, 1965); R Klein, "Spirito peregrino" in Revue d'études italiennes, 11 (1965): 197-236 (poi in La forme et l'intelligible [Parigi: Gallimard, 1970], pp. 31-64); M. Ciavolella, La 'malattia d'amore' dall'antichità al medioevo (Roma: Bulzoni, 1976), 137-41 e passim; G. Agamben, Stanze - La parola e il fantasma nella cultura occidentale (Torino: Einandi, 1977), p. 121 sgg. e passim.

8 Ars Versificatoria, 58 (ed. E. Faral, in Les Artes poètiques du XII et du XIII siècle [Parigi: H. Champion, 1958], p. 130 sg.). 